\title{
Distributed Nonlinear Model Predictive Control for Connected Autonomous Electric Vehicles Platoon with Distance-Dependent Air Drag Formulation
}

\author{
Bianca Caiazzo *,+(i), Angelo Coppola $+\left(\mathbb{D}\right.$, Alberto Petrillo ${ }^{+}\left(\mathbb{D}\right.$ and Stefania Santini ${ }^{+}(\mathbb{C})$ \\ Department of Information Technology and Electrical Engineering (DIETI), University of Naples Federico II, \\ 80125 Naples, Italy; angelo.coppola@unina.it (A.C.); alberto.petrillo@unina.it (A.P.); \\ stefania.santini@unina.it (S.S.) \\ * Correspondence: bianca.caiazzo@unina.it \\ + Authors are in alphabetic order.
}

Citation: Caiazzo, B.; Coppola, A.; Petrillo, A.; Santini, S. Distributed Nonlinear Model Predictive Control for Connected Autonomous Electric Vehicles Platoon with

Distance-Dependent Air Drag

Formulation. Energies 2021, 14, 5122.

https://doi.org/10.3390/en14165122

Academic Editor: Frede Blaabjerg

Received: 16 July 2021

Accepted: 12 August 2021

Published: 19 August 2021

Publisher's Note: MDPI stays neutral with regard to jurisdictional claims in published maps and institutional affiliations.

Copyright: () 2021 by the authors. Licensee MDPI, Basel, Switzerland. This article is an open access article distributed under the terms and conditions of the Creative Commons Attribution (CC BY) license (https:// creativecommons.org/licenses/by/ $4.0 /)$.

\begin{abstract}
This paper addresses the leader tracking problem for a platoon of heterogeneous autonomous connected fully electric vehicles where the selection of the inter-vehicle distance between adjacent vehicles plays a crucial role in energy consumption reduction. In this framework, we focused on the design of a cooperative driving control strategy able to let electric vehicles move as a convoy while keeping a variable energy-oriented inter-vehicle distance between adjacent vehicles which, depending on the driving situation, was reduced as much as possible to guarantee air-drag reduction, energy saving and collision avoidance. To this aim, by exploiting a distance-dependent air drag coefficient formulation, we propose a novel distributed nonlinear model predictive control (DNMPC) where the cost function was designed to ensure leader tracking performances, as well as to optimise the inter-vehicle distance with the aim of reducing energy consumption. Extensive simulation analyses, involving a comparative analysis with respect to the classical constant time headway $(\mathrm{CTH})$ spacing policy, were performed to confirm the capability of the DNMPC in guaranteeing energy saving.
\end{abstract}

Keywords: electric vehicles (EVs); e-platoon; distributed nonlinear model predictive control; energy consumption; air drag coefficient

\section{Introduction}

Nowadays, electrification is an effective approach towards low-carbon future transportation [1]. Indeed, since the transportation sector accounts for approximately $25 \%$ of global energy consumption and $26 \%$ of energy-related carbon dioxide emissions, the wide spread of electric vehicles (EVs) has been proven to be the most suitable environmentalfriendly choice with respect to conventional vehicles, thus achieving crucial acceptance in today's market [1,2]. Several works have highlighted the main benefits of EVs as follows (see [3,4] and references therein): (i) greenhouse gas emission reduction compared to traditional internal combustion engine vehicles (ICEVs); (ii) noise reduction, which represents a benefit for individual users, non-users (e.g., cyclists or pedestrians) and more generally, for the whole urban environment since it is considered as a new pollutant agent; (iii) improved performances with respect to ICEVs in terms of accelerations and energy efficiency due to instant torque and comfortable driving; (iv) economic saving both from an individual and a social point of view; (v) a positive coordination with renewable energy sources (RESs) within an electric grid by adjusting the load variation while offsetting the negative impact of RESs on the grid due to their intermittent nature. For all these reasons, related technologies are constantly developing with the aim to improve, as much as possible, both safety and energy saving capabilities of EVs. For instance, in the aim of regulating in real time the vehicle slip ratio toward its optimal value for the maximisation 
of the tire adhesive force, ref. [5] proposed a novel hybrid controller, called acceleration slip regulation (ASR). A robust control strategy for an in wheel motor-drive EV has been suggested in [6] to enhance vehicle lateral stability in the presence of time delays, while [7] proposed a fault-tolerant controller which, by driving the steering angle sensor of an EV, ensures the trajectory tracking.

In addition to the usage of EVs, another energy-efficient solution for transport is to operate along the road platoons of connected autonomous distributed electric vehicles (CADEVs) since they could bring many benefits in terms of driving safety and comfort, as well as traffic congestion [8]. It is well known that, when in a platoon, vehicles move in a fleet tracking a desired velocity profile (provided by the leading vehicle or by an external infrastructure, compatible with legal road constraints) while maintaining a small inter-vehicle distance so as to reduce air resistance and energy consumption [9-11]. Due to the aforementioned advantages, the platooning control problem for ICEVs has become a hot topic during recent decades in automotive and intelligent transportation research fields [12,13]. For instance, recent control solutions have been suggested in [14-17]. Specifically, a proportional-derivative controller has been suggested in [14] to address the problem of cooperative adaptive cruise control (CACC) for a connected autonomous vehicles (CAVs) platoon under a dynamic information flow topology that allows to consider communication failures, while denial-of-service (DoS) attack phenomena have also been tackled in [15] via a sampled-data diffusive control law whose exponential stability analysis is proven by exploiting time-delay system theory. Instead, the coexistence of CAVs and human-driven vehicles (HDVs) on the road has been considered in [16,17], where a new model for the mimicking of the mixed platoon has been introduced by leveraging multi-agent systems (MASs) theory and car-following model, while consensus controllers have been used to stabilise the overall mixed vehicular network. The problem of heterogeneous vehicle platoons affected by model uncertainties and external disturbances was addressed in [18], where authors developed a tube-based MPC aiming to guarantee leader-tracking purposes in the presence of spatial-geometry constraints.

Although the deployment of CADEVs will play a crucial role in eco-intelligent transportation systems, only few works explored the energy-saving benefit of an EVs platoon consisting of more than two vehicles [19-21].

In addition to considering a platoon of CADEVs with a comprehensive dynamical model to represent all the required internal components, another crucial issue to be tackled in the platooning application for energy-saving purposes is related to the choice of the intervehicle spacing policy to be imposed between adjacent vehicles. The two most commonly used spacing policies are constant spacing (CS) and the constant time headway (CTH) ones [22]. More specifically, the former sets the inter-vehicle gap as a constant value, thus reducing the platoon length and improving road throughput, while the latter defines the gap distances as a linear function of the vehicle speed, thus better miming the human drivers' behaviour [22]. Nevertheless, for complex traffic scenarios, e.g., in the presence of the sudden acceleration/deceleration of the preceding vehicle, both CS and CTH policies do not perform well [23]. To overcome this issue and to provide more flexibility with respect to the aforementioned spacing strategies, the variable time headway (VTH) policy, where the headway time is time-variant, have been introduced.

Based on these facts, we can observe that both the selection of the platoon control protocol and the selection of the spacing policy are pertinent with the aerodynamic interactions among the vehicles belonging to the platoon [24]. At steady-state operation, the aerodynamic drag coefficient depends on the specific position of each vehicle within the platoon and it is usually assumed to be known and constant. Conversely, during the transient phase associated with different manoeuvres (e.g., acceleration or braking), the aerodynamic effects result in significant variations of the air drag coefficient that should be taken into account [24]. Indeed, for each vehicle belonging to the platoon, the coefficient of the air-drag force varies as a function of the distance to the predecessor vehicle and this distance-dependent formulation should be considered from the control design phase so as 
to explicitly consider the impact of the spacing policy on aerodynamic forces, especially from an energy-saving point of view [24-26]. As such, ref. [24] addresses the longitudinal platoon control problem with a more precise modelling of the effects of the air-drag force via two $\mathcal{H}_{\infty}$ controllers able to guarantee the string stability and the achievement of smaller spacing errors without aggressive manoeuvres, respectively. The same distance-dependent air-drag formulation has been used in [25] for a heavy-duty fuel vehicle platoon with the aim of safely and fuel-efficiently coordinating its motion. Also considering the same air drag formulation, ref. [26] introduces a stochastic optimisation procedure aiming to find both controller parameters and an optimal CHT spacing policy by taking into account disturbances and transmission time delays.

From the literature overview on the platooning control, it is clear that the choice of the spacing policy plays a crucial role for guaranteeing energy-saving requirements, especially in electric vehicle platooning where the battery management strongly affects the vehicle life cycle. Therefore, avoiding energy waste is very crucial for prolonging the life-cycle of the battery [27]. Within this context, in this paper, by embedding a distance-dependent air drag formulation into the vehicle prediction model, we designed an energy-saving oriented distributed nonlinear model predictive control (DNMPC) for a heterogeneous platoon of CADEVs in order to guarantee a three-fold control objective: (i) to ensure that each vehicle tracks the leader speed profile, assumed to already be optimised in terms of energy consumption and directly or indirectly known by each vehicle within the platoon; (ii) to compute, for each time instant, the optimal variable inter-vehicle distance from the vehicle ahead by taking into account safety and road capacity constraints, as well as the electric power saving requirement and distance-dependent air-drag formulation; (iii) to guarantee the minimisation of the required battery power, thus achieving energy saving objective. Note that the proposed energy-oriented architecture computes a variable spacing policy that has a direct impact on the air drag coefficient. In so doing, based on the typical minimum and maximum values of time headway [28], inter-vehicle distance constraints are defined in order to guarantee a trade-off between smaller inter-vehicular distances, which increase the rear-end collisions risk, and larger spacing that, instead, reduces the road capacity. An extensive numerical analysis involving a comparative analysis of a typical CTH spacing policy with a discussion about the computational load confirms the benefits of the proposed control approach in ensuring energy saving.

Finally, the paper is organised as follows. Some mathematical facts are given in Section 2, while both the problem statement and the EVs modelling, along with a powerbased energy consumption estimation model, are provided in Section 3. In Section 4, the control approach is presented, while numerical results are disclosed in Section 5, where a comparison with respect to a control architecture embedding a CTH is also performed. Conclusions are drawn in Section 6.

\section{Math Preliminaries}

Leveraging the MAS framework, a set of connected vehicles can be modelled as a directed graph $\mathcal{G}_{N}=\left\{\mathcal{V}_{N}, \mathcal{E}_{N}\right\}$, with $\mathcal{V}_{N}=1,2, \ldots, N$ the set of vehicles belonging to the network, while $\mathcal{E}_{N}=\mathcal{V}_{N} \times \mathcal{V}_{N}$ is the edges set used to mimic direct and active communication links. The properties of the graph $\mathcal{G}_{N}$ can be described by three matrices, i.e., the degree matrix $\mathcal{D}$, the adjacency matrix $\mathcal{A}$ and the Laplacian matrix $\mathcal{L}$. The adjacency matrix $\mathcal{A}=\left[a_{i j}\right] \in \mathbb{R}^{N \times N}$ is such that $a_{i j}=1$ if vehicle $j$ sends information to vehicle $i$, otherwise $a_{i j}=0$. The matrix $\mathcal{D}$, instead, is defined as $\mathcal{D}=\operatorname{diag}\left\{d_{1}, d_{2}, \ldots, d_{N}\right\}$, with $d_{i}=\sum_{j=1}^{N} a_{i j}$ being the number of communication links entering in $i$. Given $\mathcal{A}$ and $\mathcal{D}$, the Laplacian matrix is defined as $\mathcal{L}=\mathcal{D}-\mathcal{A}$. Moreover, we introduce the neighbouring set $\mathcal{N}_{i}$ for the $i$-th EV as the set $\mathcal{N}_{i}=\left\{j \mid a_{i j}=1\right\}$ : this means that vehicle $i$ can receive information from any $j \in \mathcal{N}_{i}$. In a similar way, we denote with $\mathcal{O}_{i}=\left\{j \mid a_{j i}=1\right\}$ the set of agents receiving the information from node $i$. Throughout the paper, we consider $N$ EVs together with an additional one acting as a leader agent in providing the reference behaviour and indexed with 0 . Therefore, an augmented directed graph $\mathcal{G}_{N+1}$ is used to 
model the emerging communication network topology. To mimic the connection between each EV $i$ and the leader, we introduce the Pinning matrix $\mathcal{P} \in \mathcal{R}^{N \times N}$. It is defined as $\mathcal{P}=\operatorname{diag}\left\{p_{1}, p_{2}, \ldots, p_{N}\right\}$ with $p_{i}=1$ if the leading vehicle is directly connected to the $i$-th EV, otherwise $p_{i}=0$. If $p_{i}=1$, the vehicle $i$ is called pinned node. It follows that different connected communication topologies may arise which are connected, although not completely connected. In so doing, by indicating a directed path from node $i_{1}$ to node $i_{k}$ as a sequence of edges $\left(i_{1}, i_{2}\right),\left(i_{2}, i_{3}\right), \ldots,\left(i_{k-1}, i_{k}\right)$, with $\left(i_{j-1}, i_{j}\right) \in \mathcal{E}_{N}, \forall j=\{2, \ldots, k\}$, it is also possible to introduce the definition of spanning tree as a tree connecting all nodes of the graph $G_{N}$ via a directed path [29]. Throughout this work, we assume that the communication graph $\mathcal{G}_{N+1}$ is such that the following assumption holds:

Assumption 1 ([30]). $\mathcal{G}_{N+1}$ contains a directed spanning tree with the leader as the root.

This assumption allows to guarantee that each EV follower can directly or indirectly obtain leader information, thus ensuring the internal stability of the platoon. Leveraging this framework, several communication network topologies may arise, and the most common for platooning are shown in Figure 1, i.e., predecessor-following (P-F), leaderpredecessor-following (L-P-F), bidirectional-leader (B-D-L), all-to-all (broadcast, BR).

(a)

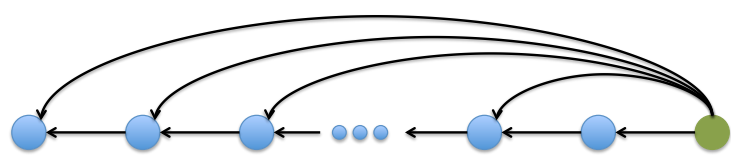

(b)

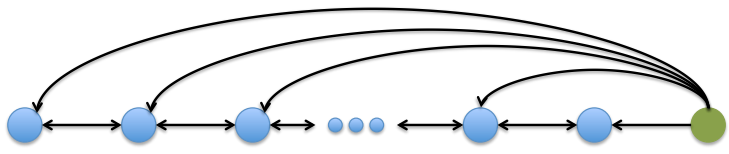

(c)

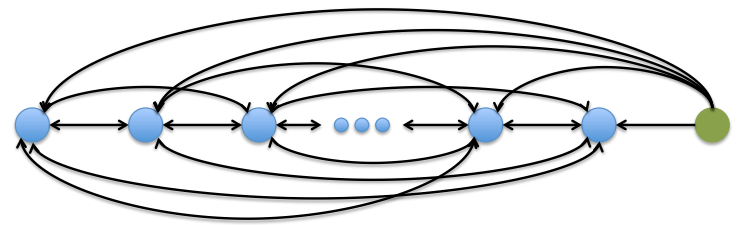

(d)

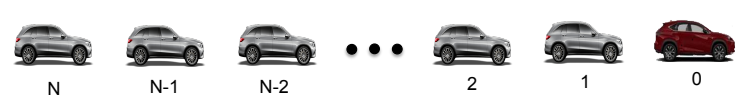

(e)

Figure 1. Exemplar communication topologies for a platoon of autonomous connected vehicles [13]: (a) predecessor-following (P-F), (b): leader-predecessor-following (L-P-F); (c): bidirectional-leader (B-D-L); (d): all-to-all (Broadcast, BR); and (e): platoon of $N$ vehicles plus a leader.

\section{E-Platoon Modelling and Control Objectives}

Consider a heterogeneous e-platoon consisting of $N$ vehicles plus an additional one, labelled as 0 , acting as a leader in providing the reference behaviour to the whole vehicular network. The platoon is arranged as a convoy, with vehicles travelling along a straight road and able to share their position, speed and acceleration information via $\mathrm{V} 2 \mathrm{~V}$ wireless communication networks (based on IEEE 802.11p communication standard or 5G communication) [31,32]. In our technological scenario, each EV is equipped with an on-board inertial sensor and a GPS receiver for measuring its state information, as well as with 
transmitting devices enabling the connectivity among vehicles within the e-platoon [33]. The aim was to guarantee that each EV tracks the leader behaviour while maintaining a safe and energy-saving oriented inter-vehicle distance with respect to the predecessor vehicle ahead. Indeed, the desired optimal gap distance is properly computed in order to reduce the energy consumption of each $i$-th vehicle within the platoon by acting on air-drag coefficient reduction, which varies as a function of inter-vehicle distance.

In what follows, we provide a detailed model of the EV by describing its nonlinear longitudinal dynamics and the battery pack model. Then, we introduce the power-based energy consumption estimation model to compute its required power for a specific drive cycle.

\subsection{Nonlinear Longitudinal EV Model}

The longitudinal behaviour of each EV $i(\forall i=1, \cdots N)$ can be depicted by the following nonlinear dynamics [34-36]:

$$
\begin{aligned}
\dot{p}_{i}(t) & =v_{i}(t) \\
\dot{v}_{i}(t) & =\frac{\eta_{i}}{R_{i} m_{i}} u_{i}(t)-g \sin \left(\theta_{i}(t)\right)-g \cos \left(\theta_{i}(t)\right) \frac{C_{r}}{1000}\left(c_{1} v(t)+c_{2}\right) \\
& -\frac{\rho}{2 m_{i}(t)} C_{D_{i}}\left(d_{i, i-1}(t)\right) A_{f_{i}} v_{i}^{2}(t),
\end{aligned}
$$

where $p_{i}(t)[m] \in \mathbb{R}$ and $v_{i}(t)(\mathrm{m} / \mathrm{s}) \in \mathbb{R}$ are the position and the speed of vehicle $i$; $u_{i}(t)(\mathrm{N} \mathrm{m})$ is the control input representing the vehicle propulsion torque; $m_{i}(\mathrm{~kg})$ is the mass of vehicle; $\eta_{i}$ is the drive-train efficiency; $R_{i}(\mathrm{~m})$ is the radius of vehicle wheel; the parameters $C_{r}, c_{1}$ and $c_{2}$ are related to the rolling resistance force and vary on basis of the road surface condition and the type of the vehicle tire; $\rho\left(\mathrm{kg} / \mathrm{m}^{3}\right)$ is the air density; $A_{f_{i}}\left(\mathrm{~m}^{2}\right)$ is the frontal area of vehicle $i ; g\left(\mathrm{~m} / \mathrm{s}^{2}\right)$ is the gravity acceleration, while $\theta_{i}(t)$ and $(\mathrm{rad})$ is the road-track slope. Furthermore, $C_{D_{i}}\left(d_{i, i-1}(t)\right)$ is the vehicle drag coefficient of vehicle $i$, which varies on the basis of the distance with respect to the ahead $(i-1)$ vehicle as [26]:

$$
C_{D_{i}}\left(d_{i, i-1}(t)\right)=C_{a}\left(1-\frac{C_{b}}{C_{c}+d_{i, i-1}(t)}\right),
$$

where $C_{a}$ is the $i$-th vehicle air-drag coefficient in the absence of any slipstream, i.e., it represents the leading vehicle air-drag coefficient, while $C_{b}$ and $C_{c}$ are positive constants whose values have been experimentally found in [37]. Note that drag coefficient formulation as in (2) takes into account the fact that the air-drag force is strictly related to both the vehicle shape and air flow around it. Indeed, the aerodynamic resistance depends on how quickly and uniformly the air cut by the vehicle rejoins the vehicle downstream, i.e., the turbulence level and wake shape. This implies that when the shape of a vehicle is streamlined or a vehicle follows another one at a closer spacing, the aerodynamic resistance is lower (see [38] and references therein). Moreover, some experimental works on the aerodynamic interactions among the vehicles in convoys are presented in the technical literature about automated highway systems $[39,40]$ and the air-drag coefficients are founded based upon them. By exploiting state space formalism and by introducing the state vector for the vehicle $i$ as $x_{i}(t)=\left[p_{i}(t), v_{i}(t)\right] \in \mathbb{R}^{2 \times 1}$, the nonlinear system in (1) $\forall i \in\{1, \ldots, N\}$ can be re-written in a more compact notation as [35]

$$
\dot{x}_{i}(t)=\left[\begin{array}{c}
v_{i}(t) \\
\varphi_{i}\left(v_{i}(t)\right)
\end{array}\right]+\left[\begin{array}{c}
0 \\
b_{i}
\end{array}\right] u_{i}(t)
$$


where $b_{i}=\eta_{i} /\left(m_{i} R_{i}\right)$, while $\varphi_{i}\left(v_{i}(t)\right) \in \mathbb{R}$ is nonlinear vector field, assumed to be bounded, continuous and differentiable. On the other hand, the leader dynamics acting as a reference for the whole vehicular network is described by the following autonomous nonlinear system:

$$
\dot{x}_{0}(t)=\left[\begin{array}{c}
v_{0}(t) \\
\varphi_{0}\left(v_{0}(t)\right)
\end{array}\right]
$$

where $x_{0}(t)=\left[p_{0}(t), v_{0}(t)\right] \in \mathbb{R}^{2 \times 1}$ are the leader state vector, with $p_{0}(t)(\mathrm{m})$ and $v_{0}(t)$ $(\mathrm{m} / \mathrm{s})$ its position and speed, respectively.

\subsection{Battery Pack Model}

To model the battery pack of each vehicle $i$ within the platoon, according to [1], we consider an equivalent simplified electric circuit consisting of a voltage supply $E_{o c, i}, 2$ ideal diodes, and 2 internal resistances $R_{i n, i}^{+}$and $R_{i n, i}^{-}$, representing the charging and discharging inner battery pack resistances, whose values depend on the battery state of charge (SOC) actual value. By defining the voltage at the terminal of the battery as

$$
V_{t, i}=E_{o c, i}-R I_{b a t t, i}= \begin{cases}E_{o c, i}-R_{i n, i}^{+} I_{b a t t, i} & \text { ifdischarging } \\ E_{o c, i}-R_{i n, i}^{-} I_{b a t t, i} & \text { ifcharging, }\end{cases}
$$

where $P_{r e q, i}$ is the related required power, the corresponding current $I_{b a t, i}(t)$ can be hence formulated as

$$
I_{b a t t, i}(t)= \begin{cases}\frac{E_{o c, i}-\sqrt{E_{o c, i}^{2}-\frac{4 R_{i n, i}^{-} P_{r e q, i}}{n_{b, i}}}}{2 R_{i n, i}^{-}} & \text {ifdischarging } \\ \frac{E_{o c, i}-\sqrt{E_{o c, i}^{2}-\frac{4 R_{i n, i}^{-} P_{r e q, i}}{n_{b, i}}}}{2 R_{i n, i}^{+}} & \text {ifcharging, }\end{cases}
$$

where $n_{b, i}$ is the number of cells constituting the battery. In so doing, we can derive the $\operatorname{SOC}_{i}(t)$ as

$$
\operatorname{SOC}_{i}(t)= \begin{cases}-\frac{1}{C_{\text {batt }, i}} \int_{0}^{t} I_{b a t t, i}(\tau) d \tau & \text { ifdischarging } \\ -\frac{\eta_{b a t t, i}}{C_{b a t t, i}} \int_{0}^{t} I_{b a t t, i}(\tau) d \tau & \text { ifcharging, }\end{cases}
$$

where $C_{b a t t, i}$ is the battery capacity, while $\eta_{b a t t, i}$ is the recharging efficiency of the battery.

\subsection{Power-Based Energy Consumption Estimation Model}

In order to deal with the estimation of the energy consumption of the $i$-th vehicle $\left(E C_{i},(\mathrm{~kW} \mathrm{~h} / \mathrm{km})\right)$, we compute the power at the electric motor $P_{e m, i}(t)$ according to the comprehensive power-based EV energy consumption model (CPEM) [41] as

$$
P_{e m, i}(t)=\left(m_{i} a_{i}(t)-\varphi_{i}\left(v_{i}(t)\right)\right) \frac{1}{\eta_{i} \eta_{e m}},
$$

where $a_{i}(t)\left(\mathrm{m} / \mathrm{s}^{2}\right)$ and $\eta_{e m}=0.91$ are the acceleration of vehicle $i$ and its electric motor efficiency, respectively, while $m_{i}, \varphi_{i}\left(v_{i}(t)\right)$ and $\eta_{i}$ were already defined in Section 3.1. Since the appraised electric vehicle is also equipped with a regenerative braking system, the effective electric power $P_{e m_{e f f, i}}(t)$ can be computed by taking into account the regenerative braking efficiency $\eta_{r b_{i}}(t)$ as follows:

$$
P_{e m_{e f f, i}}(t)= \begin{cases}P_{e m, i}(t) & \text { if } P_{e m, i}(t) \geq 0 \\ P_{e m, i}(t) \cdot \eta_{r b_{i}}(t) & \text { if } P_{e m, i}(t)<0\end{cases}
$$


Given the power consumed by the auxiliary systems of the vehicle [41], i.e., $P_{a u x}$, the electric power $P_{r e q, i}(t)$ required by vehicle $i$ is given as

$$
P_{\text {req }, i}(t)=P_{e m_{e f f, i}}(t)+P_{a u x} .
$$

Finally, from Equations (8)-(10), the EC of the vehicle $i$ is finally computed as

$$
E C_{i}=\frac{1}{3600000} \int_{0}^{t} P_{r e q, i}(\tau) d \tau \times \frac{1}{D_{i}},
$$

where $D_{i}(\mathrm{~km})$ is the travelled distance by the $i$-th vehicle.

\section{Design of Distributed Distance-Based Nonlinear Model Predictive Control}

The aim of e-platoon control is to guarantee that each EV $i(\forall i=1, \ldots, N)$, in a distributed fashion, tracks the leading vehicle's dynamics, which provides an optimal reference behaviour guaranteeing the safety and the energy saving requirements by explicitly taking into account air-drag reduction due to the presence of distance-dependent air drag coefficient $C_{D_{i}}\left(d_{i, i-1}(t)\right)$. More specifically, our objective was to design a distributed controller $u_{i}(t)$ in (3) for each vehicle $i$ such that $\forall i \in\{1, \ldots, N\}$ :

$$
\begin{aligned}
& \lim _{t \rightarrow \infty}\left\|v_{i}(t)-v_{0}(t)\right\|=0 ; \\
& \lim _{t \rightarrow \infty}\left\|p_{i}(t)-p_{0}(t)-\tilde{d}_{i, 0}(t)\right\|=0 ; \\
& u_{i}=\arg \left(\min _{u_{i}} P_{r e q, i}\left(u_{i}(t), \tilde{d}_{i, i-1}(t)\right)\right),
\end{aligned}
$$

where $\tilde{d}_{i, 0}(t)$ is the desired spacing policy between vehicle $i$ and vehicle 0 while and $\tilde{d}_{i, i-1}(t)$ is the desired one between vehicle $i$ and its predecessor $i-1$. Note that these desired safe distances are properly computed by taking into account safety constraints and without assuming a fixed time-headway value.

To fulfil the (12), we designed the energy-optimal control input $u_{i}(t)$ via a DNMPC strategy as the solution of the following constrained multiple optimisation problem.

Problem $\mathcal{F}_{i}$ : Let Assumption 1 hold. Given the optimal reference trajectory to be tracked, i.e., $x_{0}(t)$, and the information sent by the neighbouring vehicles $\mathcal{N}_{i}$, for each vehicle $i$, we find $u_{i}(t)$ such that, at each time instant $\mathrm{t}$ :

$$
\begin{gathered}
\min _{u_{i}} \mathcal{J}_{i}=\int_{t}^{t+T} L_{i}\left(x_{i}^{p}(\tau, t), x_{i}^{a}(\tau, t), x_{0}(\tau, t), x_{j}^{a}(\tau, t), \tilde{d}_{i, i-1}(\tau, t), u_{i}^{p}(\tau, t)\right) d \tau \\
\quad \text { subject to } \\
\dot{x}_{i}=f_{i}\left(x_{i}, u_{i}\right) \\
x_{i}^{p}(\tau, t)=x_{i}(t) \\
u_{i}^{p}(\tau, t)=h_{i}\left(v_{i}^{p}(t)\right) \\
v_{i, \min } \leq v_{i}(\tau, t) \leq v_{i, \max } \\
a_{i, \min } \leq a_{i}(\tau, t) \leq a_{i, \max } \\
u_{i, \min } \leq u_{i}(\tau, t) \leq u_{i, \max } \\
d_{i, i-1}^{\min }(t) \leq \tilde{d}_{i, i-1}(\tau, t) \leq d_{i, i-1}^{\max }(t)
\end{gathered}
$$

where $u_{i}$ and $u_{i}^{p}$ denote the unknown control input to be optimised and its prediction, respectively; $x_{i}^{p}(\tau, t)$ and $x_{i}^{a}(\tau, t)$ are the predicted and the assumed state of the EV $i$, respectively; $x_{j}^{a}(\tau, t)$ is the assumed state of the communicating EV $j\left(\forall j \in \mathcal{N}_{i}\right) ; h_{i}\left(v_{i}^{p}(t)\right)=$ $\frac{R_{i}}{\eta_{i}} \varphi_{i}\left(v_{i}^{p}(t)\right)$ is used to counterbalance the external forces [42]; $(\cdot)_{\max }$ and $(\cdot)_{\min }$ stand for the maximum and the minimum bounds for the related variable $(\cdot)$. More specifically, $d_{i, i-1}^{\max }(t)$ and $d_{i, i-1}^{\min }(t)$ are related to the maximum and minimum allowed inter-vehicle 
distances between vehicle $i$ and its predecessor. In order to ensure emergency braking manoeuvres, as well as air drag reduction, they can be computed considering the minimum and maximum value of vehicle time-headway as [43]

$$
d_{i, i-1}^{\min }=d_{s t}+h_{\min } v_{i}(t), \quad d_{i, i-1}^{\max }=d_{s t}+h_{\max } v_{i}(t),
$$

where $d_{s t}(\mathrm{~m})$ is the standstill distance, while $h_{\min }(\mathrm{s})$ and $h_{\max }$ (s) are the lower and upper time-headway, commonly selected as $h_{\min }=0.4$ (s) and $h_{\max }=1$ (s) [28].

The integral part of the cost function $\mathcal{J}_{i}$ in (15), i.e., $L_{i}$, is here designed as

$$
L_{i}=\omega_{1} L_{i, 1}+\omega_{2} L_{i, 2}+\omega_{3} L_{i, 3}+\omega_{4} L_{i, 4}+\omega_{5} L_{i, 5}+\omega_{6} L_{i, 6}+\omega_{7} L_{i, 7}
$$

where $\omega_{1}, \omega_{2}, \omega_{3}, \omega_{4}, \omega_{5}, \omega_{6}, \omega_{7}$ are the positive weights to be properly selected and

$$
\begin{aligned}
& L_{i, 1}=\left(p_{i}^{p}(t)-p_{0}(t)-\tilde{d}_{i, 0}(t)\right)^{2} \\
& L_{i, 2}=\left(v_{i}^{p}(t)-v_{0}(t)\right)^{2} \\
& L_{i, 3}=\sum_{j \in \mathcal{N}_{i}}\left(p_{i}^{p}(t)-p_{j}^{a}(t)-\tilde{d}_{i, j}(t)\right)^{2} \\
& L_{i, 4}=\sum_{j \in \mathcal{N}_{i}}\left(v_{i}^{p}(t)-v_{j}^{a}(t)\right)^{2} \\
& L_{i, 5}=\left(x_{i}^{p}(t)-x_{i}^{a}(t)\right)^{2} \\
& L_{i, 6}=\left(u_{i}^{p}(t)-h_{i}\left(v_{i}(t)\right)\right)^{2}, \\
& L_{i, 7}=P_{\text {req }, i}\left(t, u_{i}(t), \tilde{d}_{i, i-1}(t)\right)+P_{\text {aux }} .
\end{aligned}
$$

Note that $L_{i, 1}$ and $L_{i, 2}$ in (16a) and (16b) guarantee that the $i$-th EV tracks the leader behaviour, with $L_{i, 1} \neq 0$ and $L_{i, 2} \neq 0$ if and only if $p_{i}=1$, where $p_{i}$ is the pinning matrix element defined in Section 2; conversely if $p_{i}=0$, then the $i$-th EV was unable to directly know the leader behaviour so that $L_{i, 1}=L_{i, 2}=0 . L_{i, 3}$ and $L_{i, 4}$ in (16c) and (16d) ensure that the $i$-th EV tries to reach a coordination with the assumed trajectory of the $j$-th communicating EV, and hence, $L_{i, 3} \neq 0$ and $L_{i, 4} \neq 0$ for all $j \in \mathcal{N}_{i}$, where $\mathcal{N}_{i}$ is the set of neighbours of the $i$-th EV as defined in Section 2. Term $L_{i, 5}$ in (16e) weights the deviations of the $i$-th EV state trajectories with respect to the corresponding assumed state, which is its shifted last-step optimal state and sent to the EVs belonging to the set $\mathcal{O}_{i} ; L_{i, 6}$ (16f) counterbalances the deviations of the input error from the equilibrium, according to [42]. Finally, $L_{i, 7}$ in $(16 \mathrm{~g})$ ensures the minimisation of the instantaneous power consumption.

We highlight that the DNMPC allows emulating the typical attitude of a driver by preestimating the trajectory that each EV has to maintain for a defined horizon [44]. In addition to the advantages of this kind of controller, it is fundamental to choose a proper plant model in order to ensure coherence between reality and simulations, hence obtaining correct and effective results. However, more detailed vehicle models describing, for instance, both longitudinal and lateral dynamics, as well as the interaction forces $[45,46]$ require more computational resources and efforts. In this case, the requirement of high-performances architectures arises to deal with this kind of problem (see, e.g., [44]).

Note that, since we do not fix an expression to spacing policy, this procedure allows us to embed within our architecture an energy-oriented variable spacing policy which, unlike the classical CTH (with a typical value of $h=0.8$ (s) [43]), guarantees the minimisation of the inter-vehicle distance between two adjacent EVs while satisfying safety requirements. This results in an air drag reduction and hence, in energy consumption improvement. Indeed, in so doing, for each time instant, the inter-vehicle gap distance between the $i$-th 
and its $i-1$-th predecessor is found according to constraints in (14), while ensuring the power optimisation as well as the air drag reduction.

To better disclose the advantages of exploiting this kind of energy-oriented spacing policy within our control design, we compare in Figure 2 the classical CTH spacing policy for the $i$-th EV with the proposed one. Herein, $p_{i}\left(t^{-}\right)$and $p_{i-1}\left(t^{-}\right)$are the positions of the $i$-th and $i-1$ EVs at time instant $t^{-}$, while $\tilde{p}_{i}\left(t^{+}\right)$and $\tilde{p}_{i-1}\left(t^{+}\right)$are the position of $i$-th and $i-1$ EVs at time instant $t^{+}$by embedding them with our DNMPC. Instead, $\bar{p}_{i-1}\left(t^{+}\right)$is the ideal position of the EV $i-1$ at time $t^{+}$under the CTH spacing policy with $h=0.8$ (s). Hence, two inter-vehicle distances can be considered: $\tilde{d}_{i, i-1}$ and $\bar{d}_{i, i-1}$, consisting of a common part, i.e., $d_{s t}$, plus an additional term that depends on the time-headway value. As it is possible to observe, the suggested energy-oriented strategy permits to select, whenever it is possible, a value for time-headway that is lower than the one commonly pre-fixed with a typical CTH spacing policy. Of course, this reduction strongly affects the energy saving through the distance-dependent air drag coefficient formulation.

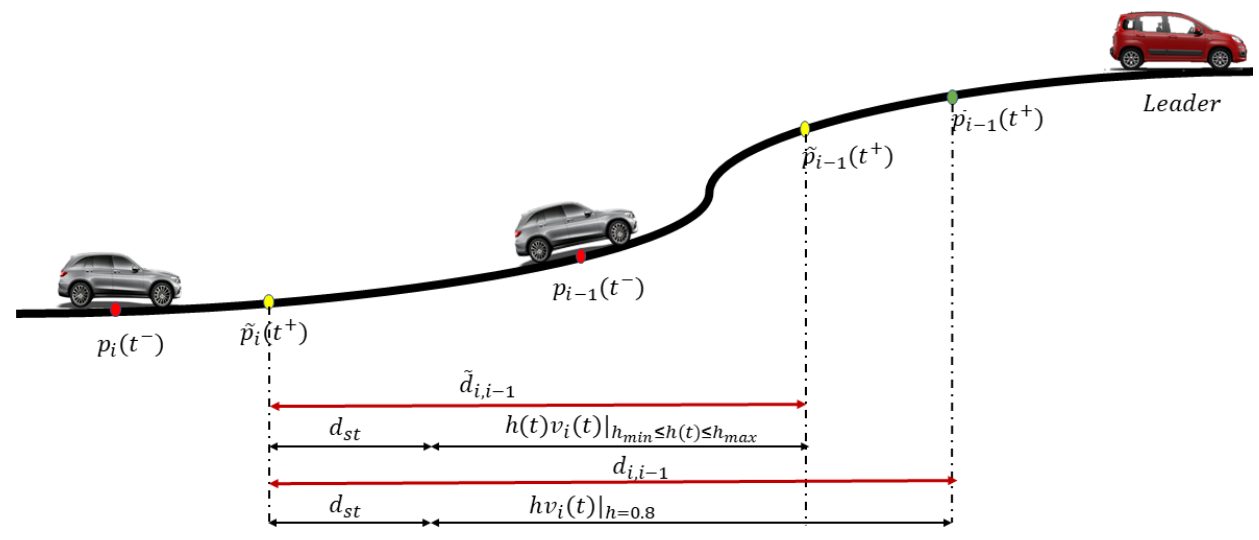

Figure 2. Energy-oriented optimal spacing policy for the EV $i$.

\section{Case Study}

To show the effectiveness of our DNMPC in (13), we consider an exemplar heterogeneous e-platoon consisting of $N=5 \mathrm{EVs}$ plus a leader moving along a flat road highway segment and connected via a LPF topology (see Figure 1). According to Section 2, the communication topology is described by leveraging graph theory, thus obtaining a static graph whose characteristic matrices are:

$$
\mathcal{P}=\left[\begin{array}{lllll}
1 & 0 & 0 & 0 & 0 \\
0 & 1 & 0 & 0 & 0 \\
0 & 0 & 1 & 0 & 0 \\
0 & 0 & 0 & 1 & 0 \\
0 & 0 & 0 & 0 & 1
\end{array}\right], \quad \mathcal{L}=\left[\begin{array}{ccccc}
0 & 0 & 0 & 0 & 0 \\
-1 & 1 & 0 & 0 & 0 \\
0 & -1 & 1 & 0 & 0 \\
0 & 0 & -1 & 1 & 0 \\
0 & 0 & 0 & -1 & 1
\end{array}\right]
$$

To disclose the benefits of our architecture, we consider two simulation scenarios: (i) basic scenario, where the DNMPC embeds a CTH spacing policy, i.e., $\bar{l}_{i, i-1}=d_{s t}+$ $h v_{i}(t)$, with a constant time-headway $h=0.8$ (s); (ii) energy-oriented scenario, where the inter-vehicle distance $\tilde{d}_{i, i-1}(t)$ is online computed, for each vehicle $i$ via the solution of the optimisation problem in (13) which explicitly takes into account the energy-saving requirement, leader tracking control objectives, as well as the safety constraints (14). The aim was to evaluate how the proposed architecture, by embedding the distance safety constraints in (14), as well as distance-dependent air drag formulation as in (2), can further reduce the energy consumption with respect to the case where the pre-fixed time-headway value $h=0.8(\mathrm{~s})$ is considered.

Note that, the time-headway values for cooperative platoon systems have to be chosen as to avoid vehicles' collisions during emergency situations [47]. 
The numerical analysis was performed via the Matlab/Simulink simulation platform while the EVs' parameters are listed in Table 1. Note that the vehicle dynamics parameters are the one of a typical passenger car and they are selected according to [48]. The battery pack parameters, as well as the electric motor efficiency are related to a Nissan leaf-type electric vehicle and they are chosen according to [1]. The leader initial state is set to $p_{0}(0)=2000(\mathrm{~m})$, while its energy-optimal speed profile, which has to be imposed to the whole vehicular network, is assumed to be known and it is highlighted in Figure 3a. Moreover, in both scenarios, the prediction horizon in Problem $\mathcal{F}_{i}, \forall i$ is set to $N_{p}=20$, while the control horizon is $N_{c}=2$. The corresponding weights $\omega_{z}, z \in\{1, \ldots, 7\}$ in the cost functional in (15) are tuned, according to the trail and error procedure $[49,50]$, as: $\omega_{1}=10 ; \omega_{2}=10 ; \omega_{3}=20 ; \omega_{4}=20 ; \omega_{5}=0.1 ; \omega_{6}=0.1 ; \omega_{7}=10$.

Table 1. Heterogeneous nonlinear vehicles parameters.

\begin{tabular}{|c|c|c|c|c|c|c|c|c|c|c|}
\hline $\begin{array}{c}\text { Vehicle } \\
\text { ID }\end{array}$ & $\begin{array}{c}m_{i} \\
(\mathrm{~kg})\end{array}$ & $\stackrel{\eta_{i}}{(-)}$ & $\begin{array}{c}R_{i} \\
(\mathrm{~m})\end{array}$ & $\begin{array}{l}C_{a} \\
(-)\end{array}$ & $\begin{array}{l}A_{f, i} \\
\left(\mathrm{~m}^{2}\right)\end{array}$ & $\begin{array}{c}a_{\max } \\
\left(\mathrm{m} / \mathrm{s}^{2}\right)\end{array}$ & $\begin{array}{c}a_{\min } \\
\left(\mathrm{m} / \mathrm{s}^{2}\right)\end{array}$ & $\begin{array}{c}C_{\text {batt }, i} \\
\text { (Ah) }\end{array}$ & $\begin{array}{l}n_{b, i} \\
(-)\end{array}$ & $\begin{array}{c}\eta_{\text {batt }, i} \\
(-)\end{array}$ \\
\hline 0 & 1545 & 0.89 & 0.3060 & 0.28 & 2.3315 & 2.5 & -6.0 & 65 & 96 & 0.97 \\
\hline 1 & 1015 & 0.89 & 0.2830 & 0.30 & 2.1900 & 2.5 & -6.0 & 65 & 96 & 0.97 \\
\hline 2 & 1375 & 0.89 & 0.2880 & 0.24 & 2.4000 & 2.5 & -6.0 & 65 & 96 & 0.97 \\
\hline 3 & 1430 & 0.89 & 0.3284 & 0.28 & 2.4600 & 2.5 & -6.0 & 65 & 96 & 0.97 \\
\hline 4 & 1067 & 0.89 & 0.2653 & 0.29 & 2.1400 & 2.5 & -6.0 & 65 & 96 & 0.97 \\
\hline 5 & 1155 & 0.89 & 0.2880 & 0.33 & 2.0400 & 2.5 & -6.0 & 65 & 96 & 0.97 \\
\hline
\end{tabular}

Note that this choice of selecting the same weighting factors for both the appraised scenario guarantees the fairness of the comparison analysis. Indeed, in so doing, we restrict our attention on the effect that the different spacing policy can have on energy-saving requirements. Results in Figure 3b,c show the behaviour of the e-platoon in the basic scenario, in terms of speed and acceleration profiles, respectively, while the inter-vehicle distance of each vehicle within the platoon with respect to its predecessor is reported in Figure 3d.

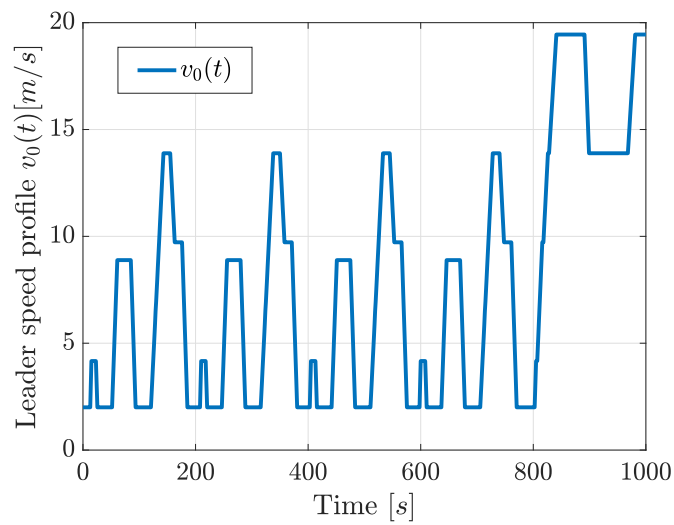

(a)

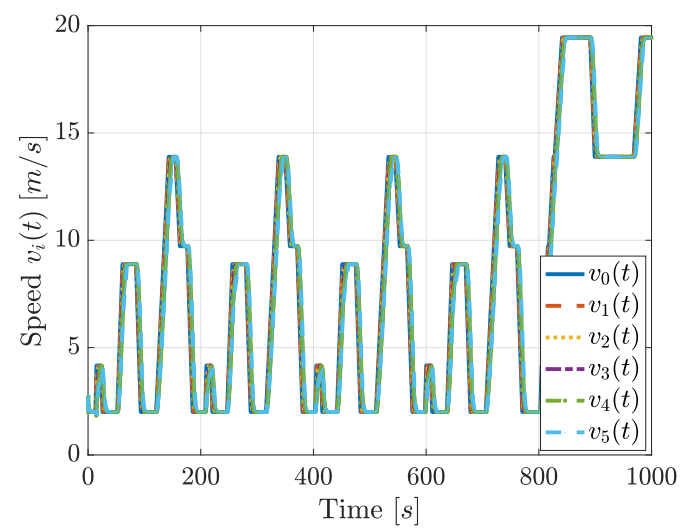

(b)

Figure 3. Cont. 


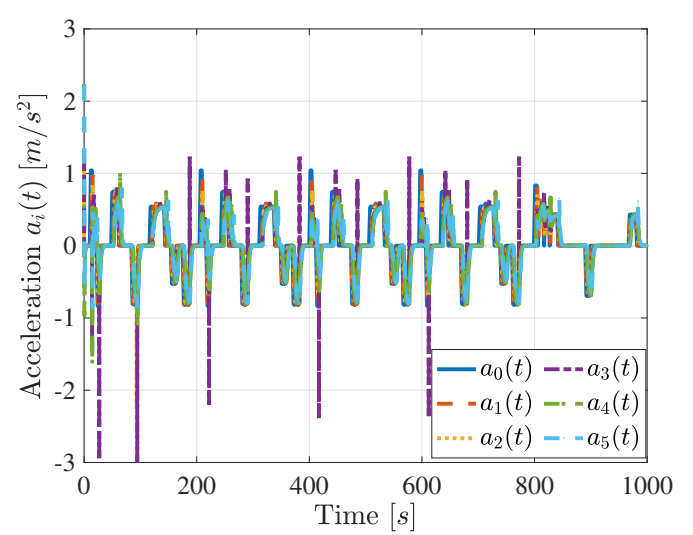

(c)

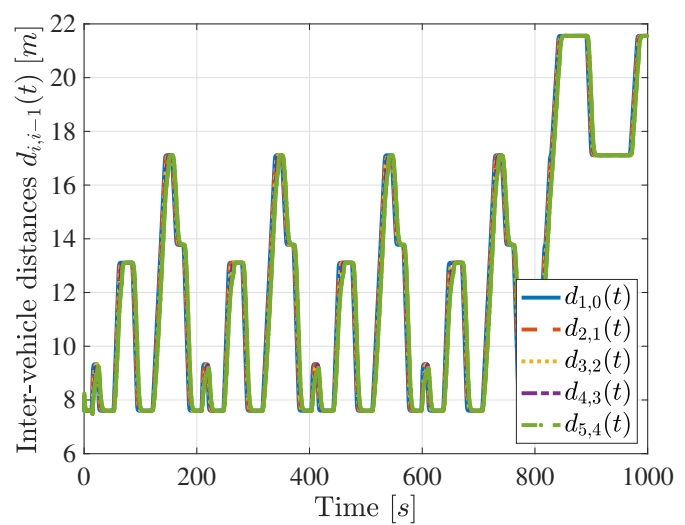

(d)

Figure 3. Basic scenario-tracking performances. Time history of: (a) energy-optimal leader speed profile; (b) vehicles speed $v_{i}(t), i=0, \ldots, 5 ;(\mathbf{c})$ vehicles acceleration $a_{i}(t), i=0, \ldots, 5$; and $(\mathbf{d})$ inter-vehicle distances $\bar{d}_{i, i-1}(t), i=1, \ldots, 5$

For the same energy-optimal leader speed profile as in Figure 3a, Figure 4 discloses the results of an energy-oriented scenario, where the inter-vehicle distances between two adjacent vehicles, properly computed by our DNMPC, is shown in Figure 4d. Good tracking performances in terms of speed and acceleration can also be appreciated in this scenario (Figure $4 \mathrm{~b}, \mathrm{c}$ ), while the time history of the longitudinal position of EVs is also shown in Figure 4a. To disclose the benefits of the proposed DNMPC, we compare the intervehicular distance obtained with our approach with respect to the basic scenario. From this perspective, Figure 4e shows the comparison between the two spacing gap distances in these two simulation scenarios, i.e., $\bar{d}_{i, i-1}(t)$ and $\tilde{d}_{i, i-1}(t)$ in basic and energy-oriented scenarios, respectively. As it is possible to observe herein, a smaller inter-vehicle gap can be achieved under the proposed energy-oriented strategy with distance-dependent air drag formulation, thus achieving better performances in terms of energy consumption. Indeed, one can find that the minimum and maximum spacing for energy-oriented scenarios are $6.4(\mathrm{~m})$ and $16.3(\mathrm{~m})$, respectively, while in the basic scenario these thresholds are $7.6(\mathrm{~m})$ and $21.5(\mathrm{~m})$, respectively. To further illustrate the benefits of the proposed energy-oriented architecture in terms of energy savings, we compute the percentage variation of energy consumption in $(\mathrm{kWh} / \mathrm{km})$ by exploiting the relation in (11). The results reported in Table 2 confirm how the proposed DNMPC guarantees an average energy reduction of $2.2 \%$ for the entire e-platoon.

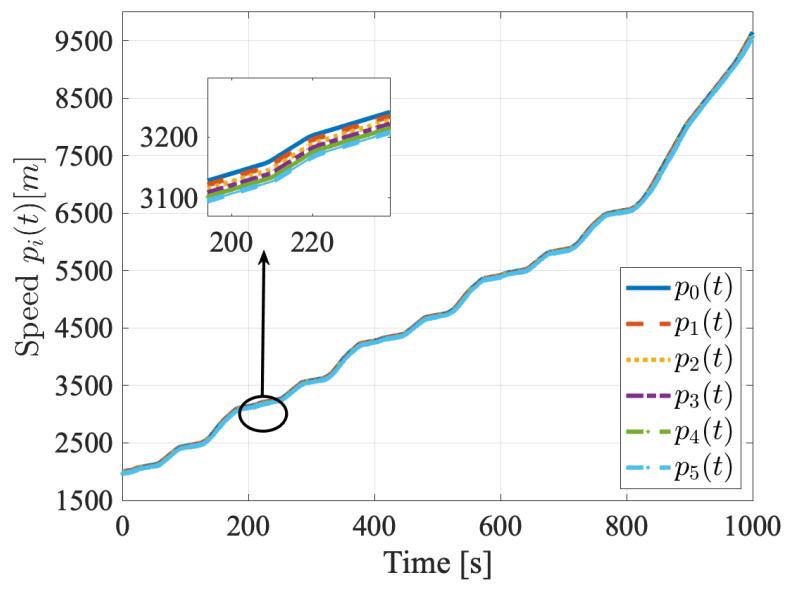

(a)

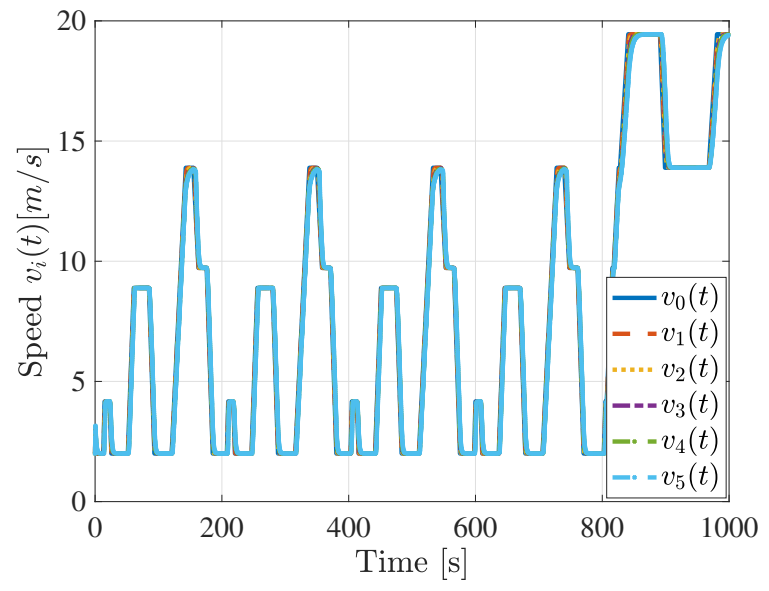

(b)

Figure 4. Cont. 


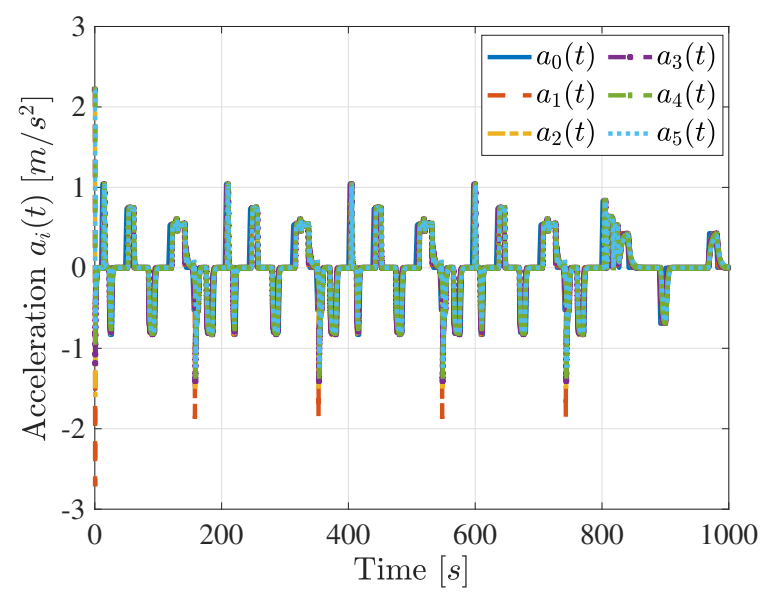

(c)

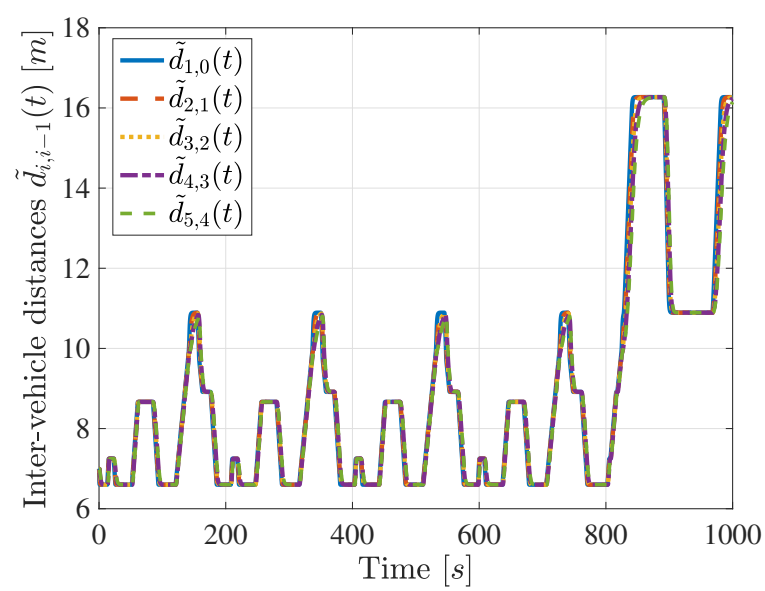

(d)

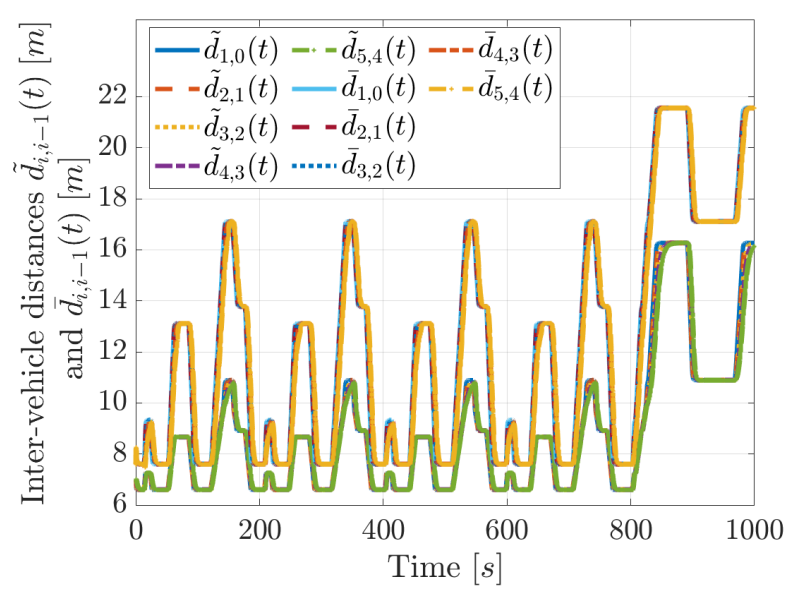

(e)

Figure 4. Energy-oriented scenario-tracking performances. Time history of: (a) vehicles longitudinal position $p_{i}(t) i=0, \ldots, 5$; (b) vehicles speed $v_{i}(t), i=0, \ldots, 5$; (c) vehicles acceleration $a_{i}(t), i=0, \ldots, 5 ;(\mathbf{d})$ inter-vehicle distances $\tilde{d}_{i, i-1}(t), i=$ $1, \ldots, 5 ;(\mathbf{e})$ comparison between inter-vehicle distances $\bar{d}_{i, i-1}(t)$ and $\tilde{d}_{i, i-1}, i=1, \ldots, 5$ in basic and energy-oriented scenarios.

Table 2. Percentage of energy saving in $(\mathrm{kWh} / \mathrm{km})$ under energy-oriented architecture with respect to the basic scenario.

\begin{tabular}{ccccccc}
\hline Configuration & Vehicle 1 & Vehicle 2 & Vehicle 3 & Vehicle 4 & Vehicle 5 & Mean \\
\hline Energy-oriented strategy & -2.1220 & -2.1971 & -2.2549 & -2.2686 & -2.2150 & -2.2115 \\
\hline
\end{tabular}

\section{DNMPC vs. Pure Diffusive Controller}

In order to highlight the benefits of our DNMPC in guaranteeing leader-tracking performances while ensuring energy saving requirements, here we also compare its performance with the one achievable via the following more classic distributed diffusive linear controller:

$$
u_{i}(t)=-K_{P} \sum_{j=0}^{N} a_{i j}\left(p_{i}(t)-p_{j}(t)-d_{i j}(t)\right)-K_{V} \sum_{j=0}^{N} a_{i j}\left(v_{i}(t)-v_{j}(t)\right),
$$

where the control gains are selected as $K_{P}=250$ and $K_{V}=220$ according to [35]; $d_{i j}(t)$ is the inter-vehicle distance between the $i$-th and $j$-th EV, properly selected exploiting the CTH spacing policy with $h=0.8$ (s). 
Our aim was to disclose that, despite the higher computational load required by the proposed control action (13), the online computation of $\tilde{d}_{i, i-1}(t)$ allows guaranteeing improved energy-saving and leader tracking performances, while complying with safety constraints. As the driving scenario, we consider the one presented in Figure 3a, while the Simulink Profiler Tool was exploited to compare the two control approaches in terms of computational load.

To this aim, we leveraged an Intel Core $\mathrm{i} 5-8300 \mathrm{H}$ processor, while the GPU is the Nvidia GeForce GTX.

The Pie-charts in Figure 5 summarise the simulation profile reports for the strategies in comparison, while the required total computational times are 572.9731 (s) and 24.057 (s) for the DNMPC approach and for (18), respectively. Specifically, for both cases, based on these total times, we disclose in Figure 5 the time percentage required by each distributed control action $u_{i}(t), \forall i \in\{1, \ldots, N\}$, while the label Other represents the residual computational load requested by the other software parts of the simulation scheme. This analysis clearly reveals the high computational burden required by the DNMPC with respect to a more classical consensus-based controllers as in (18). However, even if these latter ask for a less computational cost, they suffer from the problem of managing multiple variable constraints [44], such as the energy-saving ones appraised in this work.

Indeed, although (18) ensures good tracking leader performances (see Figure 6a), the inter-vehicle distance $d_{i, i-1}(t)$ and the diffusive controller that the vehicles maintain is greater than $\tilde{d}_{i, i-1}(t)$, ensured by the DNMPC (see Figure $6 \mathrm{~b}, \mathrm{c}$ ). This implies that the proposed energy-oriented strategy with a distance-dependent drag coefficient allows to achieve improved performances in terms of energy consumption, with an average energy reduction of $3.1204 \%$ for the entire e-platoon (see Table 3). Finally, we highlight that the higher computational load required by the DNMPC does not preclude its real-time implementation. Indeed, there exists an extensive research line devoted to this crucial aspect and different effective solutions have been found (see, e.g., [51] and references therein).

Table 3. Percentage of energy saving in $(\mathrm{kWh} / \mathrm{km})$ under energy-oriented architecture with respect to the basic scenario with the proportional controller.

\begin{tabular}{ccccccc}
\hline Configuration & Vehicle 1 & Vehicle 2 & Vehicle 3 & Vehicle 4 & Vehicle 5 & Mean \\
\hline Energy-oriented strategy & -3.6553 & -3.3454 & -3.0981 & -2.8665 & -2.6365 & -3.1204 \\
\hline
\end{tabular}
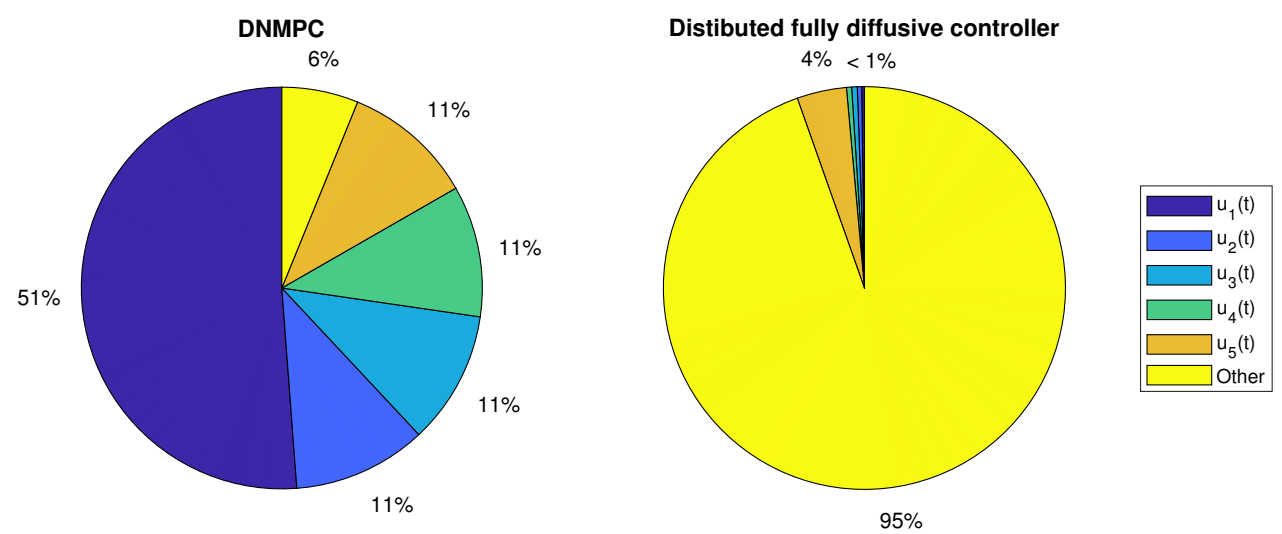

Figure 5. Computational load in energy-oriented scenario and basic-scenario with controller (18). 


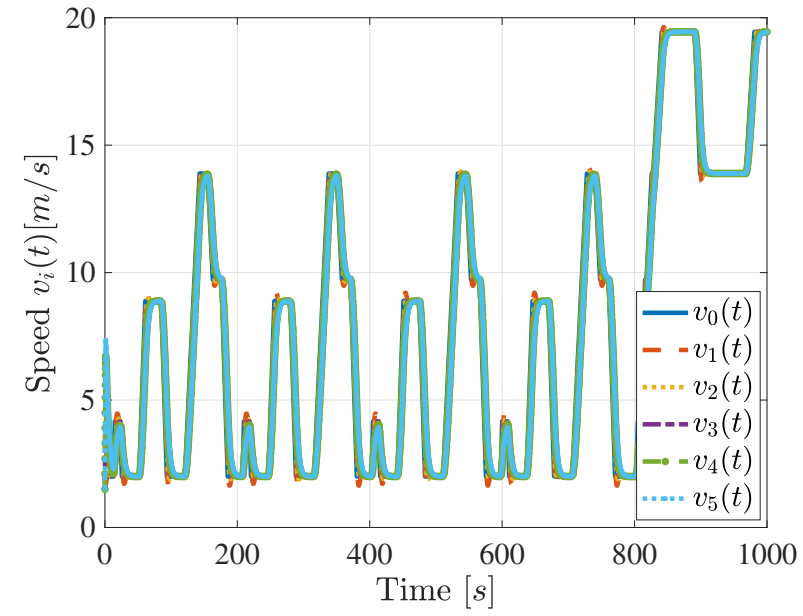

(a)

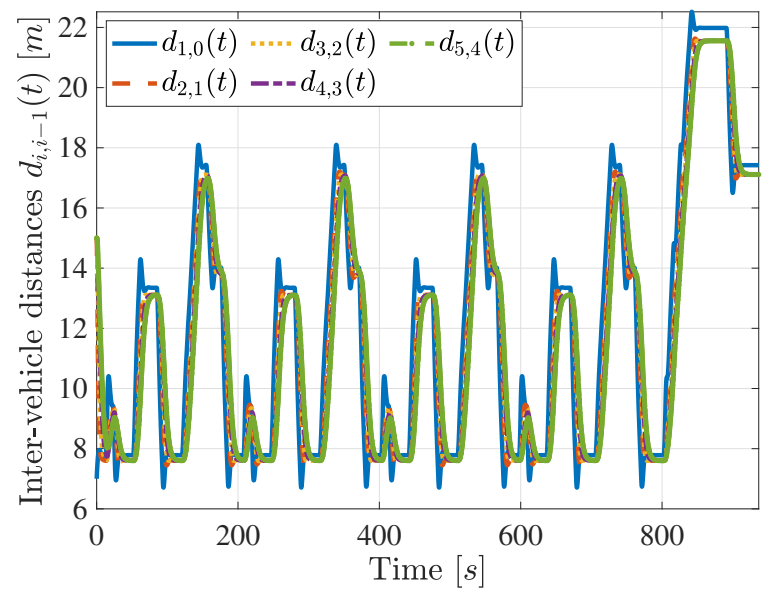

(b)

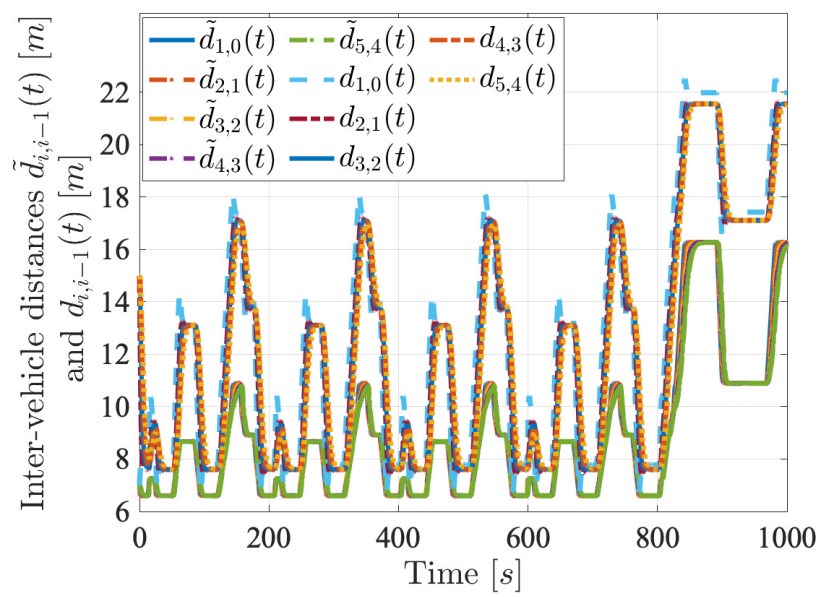

(c)

Figure 6. Tracking performances with the more classic distributed diffusive control in (18). Time history of: (a) vehicles speed $v_{i}(t), i=0, \ldots, 5 ; i=0, \ldots, 5 ;(\mathbf{b})$ inter-vehicle distances $d_{i, i-1}(t), i=1, \ldots, 5 ;(\mathbf{c})$ comparison between inter-vehicle distances $d_{i, i-1}(t), i=1, \ldots, 5$ and $\tilde{d}_{i, i-1}(t), i=1, \ldots, 5$ in basic and energy-oriented scenarios.

\section{Conclusions}

In this paper, the energy-saving and leader-tracking control problem for an heterogeneous platoon of CADEVs with nonlinear dynamics was explored and solved through a distributed nonlinear model predictive control, where the cost function for each vehicle was properly chosen according to both leader-tracking and power minimisation control objectives. Specifically, the energy consumption reduction was ensured via the integration of a distance-dependent air drag formulation, which, for each vehicle, varied in function of the distance from the ahead vehicle. The suggested energy-oriented control architecture allowed embedding an optimised variable spacing policy that considered distance constraints aiming to avoid both smaller and larger inter-vehicle gaps that could lead to rear-end collision risk and to road capacity reduction, respectively. Finally, numerical analyses have highlighted the effectiveness and the capability of the DNMPC in guaranteeing an improvement of energy performance for the EVs platoon with respect to a basic scenario, where the traditional CTH spacing policy is used, with an average energy saving of approximately $2.2 \%$. Future works could include: (i) an extensive validation of the DNMPC approach via a virtual testing co-simulation, i.e., the coordinated simulation of heterogeneous sub-models independently developed (interested readers may refer to [52-54] for 
further details), where not only a more detailed EV dynamic model (including low-level controllers, an electric motor model and inverter devices) is considered, but SUMO can also be exploited for reproducing the road network and realistic traffic conditions; and (ii) the experimental validation of the DNMPC via self-driving cars and leveraging real-time control architectures similar to the ones proposed in $[32,55]$.

Author Contributions: Conceptualization, B.C., A.C., A.P. and S.S.; methodology, B.C., A.C., A.P.; software, B.C., A.C., A.P.; validation,B.C., A.C., A.P.; formal analysis, B.C., A.C., A.P.; investigation, B.C., A.C., A.P.; resources, B.C., A.C., A.P.; data curation, B.C., A.C., A.P. All authors have read and agreed to the published version of the manuscript.

Funding: This research received no external funding.

Institutional Review Board Statement: Not applicable.

Informed Consent Statement: Not applicable.

Conflicts of Interest: The authors declare no conflict of interest.

1. Maia, R.; Silva, M.; Araújo, R.; Nunes, U. Electrical vehicle modeling: A fuzzy logic model for regenerative braking. Expert Syst. Appl. 2015, 42, 8504-8519. [CrossRef]

2. Lekshmi, S.; Lal Priya, P.S. Mathematical modeling of Electric vehicles-A survey. Control Eng. Pract. 2019, 92, 104138.

3. Zhang, T.; Pota, H.; Chu, C.C.; Gadh, R. Real-time renewable energy incentive system for electric vehicles using prioritization and cryptocurrency. Appl. Energy 2018, 226, 582-594. [CrossRef]

4. Noel, L.; de Rubens, G.Z.; Kester, J.; Sovacool, B.K. Beyond emissions and economics: Rethinking the co-benefits of electric vehicles (EVs) and vehicle-to-grid (V2G). Transp. Policy 2018, 71, 130-137. [CrossRef]

5. Ding, X.; Wang, Z.; Zhang, L. Hybrid Control-Based Acceleration Slip Regulation for Four-Wheel-Independently-Actuated Electric Vehicles. IEEE Trans. Transp. Electrif. 2020. [CrossRef]

6. Zhang, L.; Wang, Y.; Wang, Z. Robust lateral motion control for in-wheel-motor-drive electric vehicles with network induced delays. IEEE Trans. Veh. Technol. 2019, 68, 10585-10593. [CrossRef]

7. Zhang, L.; Wang, Z.; Ding, X.; Li, S.; Wang, Z. Fault-Tolerant Control for Intelligent Electrified Vehicles Against Front Wheel Steering Angle Sensor Faults During Trajectory Tracking. IEEE Access 2021, 9, 65174-65186. [CrossRef]

8. Guo, J.; Wang, J.; Li, K.; Luo, Y. Adaptive non-linear coordinated optimal dynamic platoon control of connected autonomous distributed electric vehicles on curved roads. IET Intell. Transp. Syst. 2020, 14, 1626-1637. [CrossRef]

9. He, X.; Wu, X. Eco-driving advisory strategies for a platoon of mixed gasoline and electric vehicles in a connected vehicle system. Transp. Res. Part D Transp. Environ. 2018, 63, 907-922. [CrossRef]

10. Petrillo, A.; Pescape, A.; Santini, S. A secure adaptive control for cooperative driving of autonomous connected vehicles in the presence of heterogeneous communication delays and cyberattacks. IEEE Trans. Cybern. 2020, 51, 1134-1149. [CrossRef]

11. Fiengo, G.; Lui, D.G.; Petrillo, A.; Santini, S.; Tufo, M. Distributed robust PID control for leader tracking in uncertain connected ground vehicles with V2V communication delay. IEEE/ASME Trans. Mechatron. 2019, 24, 1153-1165. [CrossRef]

12. Lee, W.J.; Kwag, S.I.; Ko, Y.D. The optimal eco-friendly platoon formation strategy for a heterogeneous fleet of vehicles. Transp. Res. Part D Transp. Environ. 2021, 90, 102664. [CrossRef]

13. Petrillo, A.; Salvi, A.; Santini, S.; Valente, A.S. Adaptive multi-agents synchronization for collaborative driving of autonomous vehicles with multiple communication delays. Transp. Res. Part C Emerg. Technol. 2018, 86, 372-392. [CrossRef]

14. Gong, S.; Zhou, A.; Peeta, S. Cooperative adaptive cruise control for a platoon of connected and autonomous vehicles considering dynamic information flow topology. Transp. Res. Rec. 2019, 2673, 185-198. [CrossRef]

15. Zhang, D.; Shen, Y.P.; Zhou, S.Q.; Dong, X.W.; Yu, L. Distributed secure platoon control of connected vehicles subject to DoS attack: Theory and application. IEEE Trans. Syst. Man Cybern. Syst. 2020. [CrossRef]

16. Chen, J.; Liang, H.; Li, J.; Xu, Z. A novel distributed cooperative approach for mixed platoon consisting of connected and automated vehicles and human-driven vehicles. Phys. A Stat. Mech. Appl. 2021, 573, 125939. [CrossRef]

17. Di Vaio, M.; Fiengo, G.; Petrillo, A.; Salvi, A.; Santini, S.; Tufo, M. Cooperative shock waves mitigation in mixed traffic flow environment. IEEE Trans. Intell. Transp. Syst. 2019, 20, 4339-4353. [CrossRef]

18. Luo, Q.; Nguyen, A.T.; Fleming, J.; Zhang, H. Unknown Input Observer Based Approach for Distributed Tube-Based Model Predictive Control of Heterogeneous Vehicle Platoons. IEEE Trans. Veh. Technol. 2021, 70, 2930-2944. [CrossRef]

19. Ma, F.; Yang, Y.; Wang, J.; Liu, Z.; Li, J.; Nie, J.; Shen, Y.; Wu, L. Predictive energy-saving optimization based on nonlinear model predictive control for cooperative connected vehicles platoon with V2V communication. Energy 2019, 189, 116120. [CrossRef]

20. Xu, L.; Zhuang, W.; Yin, G.; Bian, C. Energy-oriented cruising strategy design of vehicle platoon considering communication delay and disturbance. Transp. Res. Part C Emerg. Technol. 2019, 107, 34-53. [CrossRef] 
21. Ma, F.; Yang, Y.; Wang, J.; Li, X.; Wu, G.; Zhao, Y.; Wu, L.; Aksun-Guvenc, B.; Guvenc, L. Eco-driving-based cooperative adaptive cruise control of connected vehicles platoon at signalized intersections. Transp. Res. Part D Transp. Environ. 2021, $92,102746$. [CrossRef]

22. Bian, Y.; Zheng, Y.; Ren, W.; Li, S.E.; Wang, J.; Li, K. Reducing time headway for platooning of connected vehicles via V2V communication. Transp. Res. Part C Emerg. Technol. 2019, 102, 87-105. [CrossRef]

23. Chen, J.; Liang, H.; Li, J.; Lv, Z. Connected Automated Vehicle Platoon Control With Input Saturation and Variable Time Headway Strategy. IEEE Trans. Intell. Transp. Syst. 2020, 22, 4929-4940. [CrossRef]

24. Köroğlu, H.; Mirzaei, M.; Falcone, P.; Krajnović, S. Platoon Control Under a Novel Leader and Predecessor Following Scheme with the Use of an Advanced Aerodynamic Model. J. Dyn. Syst. Meas. Control 2018, 140, 041006 [CrossRef]

25. Turri, V. Look-Ahead Control for Fuel-Efficient and Safe Heavy-Duty Vehicle Platooning. Ph.D. Thesis, KTH Royal Institute of Technology, Stockholm, Sweden, 2018.

26. Wasserburger, A.; Schirrer, A.; Hametner, C. Stochastic Optimization for Energy-Efficient Cooperative Platooning. In Proceedings of the 2019 IEEE Vehicle Power and Propulsion Conference (VPPC), Hanoi, Vietnam, 14-17 October 2019; pp. 1-6.

27. Tie, S.F.; Tan, C.W. A review of energy sources and energy management system in electric vehicles. Renew. Sustain. Energy Rev. 2013, 20, 82-102. [CrossRef]

28. Yanakiev, D.; Kanellakopoulos, I. Nonlinear spacing policies for automated heavy-duty vehicles. IEEE Trans. Veh. Technol. 1998, 47, 1365-1377. [CrossRef]

29. Lui, D.G.; Petrillo, A.; Santini, S. An optimal distributed PID-like control for the output containment and leader-following of heterogeneous high-order multi-agent systems. Inf. Sci. 2020, 541, 166-184. [CrossRef]

30. De Tommasi, G.; Lui, D.G.; Petrillo, A.; Santini, S. AL 2-gain robust PID-like protocol for time-varying output formationcontainment of multi-agent systems with external disturbance and communication delays. IET Control Theory Appl. 2021, 15, 1169-1184. [CrossRef]

31. Coelingh, E.; Solyom, S. All aboard the robotic road train. IEEE Spectr. 2012, 49, 34-39. [CrossRef]

32. Castiglione, L.M.; Falcone, P.; Petrillo, A.; Romano, S.P.; Santini, S. Cooperative intersection crossing over 5G. IEEE/ACM Trans. Netw. 2020, 29, 303-317. [CrossRef]

33. Ryu, J.; Gerdes, J.C. Integrating inertial sensors with global positioning system (GPS) for vehicle dynamics control. J. Dyn. Sys. Meas. Control 2004, 126, 243-254. [CrossRef]

34. Wu, Y.; Li, S.E.; Cortés, J.; Poolla, K. Distributed sliding mode control for nonlinear heterogeneous platoon systems with positive definite topologies. IEEE Trans. Control Syst. Technol. 2019, 28, 1272-1283. [CrossRef]

35. Manfredi, S.; Petrillo, A.; Santini, S. Distributed PI Control For Heterogeneous Nonlinear Platoon of Autonomous Connected Vehicles. IFAC-PapersOnLine 2020, 53, 15229-15234. [CrossRef]

36. Pariota, L.; Coppola, A.; Di Costanzo, L.; Di Vico, A.; Andolfi, A.; D’Aniello, C.; Bifulco, G.N. Integrating tools for an effective testing of connected and automated vehicles technologies. IET Intell. Transp. Syst. 2020, 14, 1025-1033. [CrossRef]

37. Hucho, W.; Sovran, G. Aerodynamics of road vehicles. Annu. Rev. Fluid Mech. 1993, 25, 485-537. [CrossRef]

38. Hussein, A.A.; Rakha, H.A. Vehicle platooning impact on drag coefficients and energy/fuel saving implications. arXiv 2020, arXiv:2001.00560.

39. Zabat, M.; Stabile, N.; Farascaroli, S.; Browand, F. The Aerodynamic Performance of Platoons: A Final Report; California Department of Transportation PATH PROGRAM, University of California: Berkeley, CA, USA, 1995.

40. Mirzaei, M.; Krajnović, S. Numerical Simulation of Two Vehicles at Short Distances in a Platoon. In Proceedings of the First International Conference in Numerical and Experimental Aerodynamics of Road Vehicles and Trains (Aerovehicles 1), Bordeaux, France, 23-25 June 2014; pp. 23-25.

41. Fiori, C.; Ahn, K.; Rakha, H.A. Power-based electric vehicle energy consumption model: Model development and validation. Appl. Energy 2016, 168, 257-268. [CrossRef]

42. Zheng, Y.; Li, S.E.; Li, K.; Borrelli, F.; Hedrick, J.K. Distributed model predictive control for heterogeneous vehicle platoons under unidirectional topologies. IEEE Trans. Control Syst. Technol. 2016, 25, 899-910. [CrossRef]

43. Wu, C.; Xu, Z.; Liu, Y.; Fu, C.; Li, K.; Hu, M. Spacing policies for adaptive cruise control: A survey. IEEE Access 2020, 8, 50149-50162. [CrossRef]

44. Cosimi, F.; Dini, P.; Giannetti, S.; Petrelli, M.; Saponara, S. Analysis and Design of a Non-linear MPC Algorithm for Vehicle Trajectory Tracking and Obstacle Avoidance. In Proceedings of the International Conference on Applications in Electronics Pervading Industry, Environment and Society, Virtual, 19-20 November 2020; pp. 229-234.

45. Siampis, E.; Velenis, E.; Gariuolo, S.; Longo, S. A real-time nonlinear model predictive control strategy for stabilization of an electric vehicle at the limits of handling. IEEE Trans. Control Syst. Technol. 2017, 26, 1982-1994. [CrossRef]

46. Jazar, R.N. Vehicle Dynamics: Theory and Application; Springer: Berlin/Heidelberg, Germany, 2017.

47. Nowakowski, C.; O'Connell, J.; Shladover, S.E.; Cody, D. Cooperative adaptive cruise control: Driver acceptance of following gap settings less than one second. In Proceedings of the Human Factors and Ergonomics Society Annual Meeting, San Francisco, CA, USA, 27 September-1 October 2010; SAGE Publications: Los Angeles, CA, USA, 2010; Volume 54, pp. $2033-2037$.

48. Li, K.; Gao, F.; Li, S.E.; Zheng, Y.; Gao, H. Robust cooperation of connected vehicle systems with eigenvalue-bounded interaction topologies in the presence of uncertain dynamics. Front. Mech. Eng. 2018, 13, 354-367. [CrossRef]

49. Alhajeri, M.; Soroush, M. Tuning guidelines for model-predictive control. Ind. Eng. Chem. Res. 2020, 59, 4177-4191. [CrossRef] 
50. Ali, E. Heuristic on-line tuning for nonlinear model predictive controllers using fuzzy logic. J. Process Control 2003, 13, 383-396. [CrossRef]

51. Burk, D.; Völz, A.; Graichen, K. A modular framework for distributed model predictive control of nonlinear continuous-time systems (GRAMPC-D). Optim. Eng. 2021, 1-25. [CrossRef]

52. Bernardeschi, C.; Dini, P.; Domenici, A.; Palmieri, M.; Saponara, S. Formal Verification and Co-Simulation in the Design of a Synchronous Motor Control Algorithm. Energies 2020, 13, 4057. [CrossRef]

53. Chen, W.; Jacobson, B.J.; Ran, S. Design of interface in co-simulation for electric power assisted steering system development. In Proceedings of the 14th International Symposium on Advanced Vehicle Control, Beijing, China, 16-20 July 2018.

54. De Souza, E.; Ardakanian, O.; Nikolaidis, I. A Co-simulation Platform for Evaluating Cyber Security and Control Applications in the Smart Grid. In Proceedings of the ICC 2020-2020 IEEE International Conference on Communications (ICC), Dublin, Ireland, 7-11 June 2020; pp. 1-7.

55. Di Vaio, M.; Falcone, P.; Hult, R.; Petrillo, A.; Salvi, A.; Santini, S. Design and experimental validation of a distributed interaction protocol for connected autonomous vehicles at a road intersection. IEEE Trans. Veh. Technol. 2019, 68, 9451-9465. [CrossRef] 